\section{BUTTON BATTERY INGESTION AND INSERTION BY CHILDREN-AN INTERNATIONAL APPROACH TO AN EMERGING DANGEROUS INJURY}

doi:10.1136/injuryprev-2012-040590i.7

Eric Chalmers. Kidsafe ACT, PO Box 351, Mawson, ACT, 2607 AustraliaChair, Network Advisory Council, Safe Kids Worldwide

The Seminar will include a variety of presenters, on behalf of

- Product safety regulators-USA, Australia, New Zealand, Korea, Japan

- Community organisations-Safe Kids Worldwide, Safe Kids USA, Safe Kids Canada, Safekids NZ, Kidsafe in Australia

- Paediatricians will outline the nature and consequences of ingestion or insertion

- Research-Queensland Injury Surveillance Unit; Safe Kids Worldwide and Fleishman; Australian Competition and Consumer Commission

Each presenter will outline a brief summary of issues and implications from their work in the area.

Background Extensive cooperative work is being undertaken internationally at regulatory, community and industry levels to address this emerging cause of severe injury. This, like many developing issues, is complex. The problem arises from the misuse of batteries (often unnoticed) by young children who were never intended to have access to them; community awareness is very low; data is difficult and misdiagnosis easy; the result can be catastrophic and deadly; containing the risk is complex.

Aims/Objectives The Seminar will address all three themes for the Conference and provide a round table discussion on the issue; lessons from work to date; and next steps in addressing the trends internationally-to synthesise the issue and consider the implications for ongoing efforts.

\section{Structure of Seminar}

- Introduction and outline

- Outline of the Battery Controlled Programme and history

- Input and implications from research

- A medical profession perspective

- Industry issues and actions

- Community awareness campaign

- Regulatory responses

- Summary and conclusion

Results The Seminar will demonstrate

- How Governments, the medical profession, researchers, community groups and industry are working together to find practical solutions to the issue and benefits of this approach;

- A variety of research issues; work to overcome these; and how research has been put to immediate use in the Programme;

- Development of a successful awareness Programme and how this has started to be transported across countries and cultures;

- The process of addressing complex regulatory and standards issues impacting many areas.

Significance The Seminar' significance lies in

- Demonstration of the capacity to address emerging issues quickly when regulators, community organisations, researchers and industry work together

- An international opportunity to consider progress in addressing this emerging risk to children and the key next steps. 